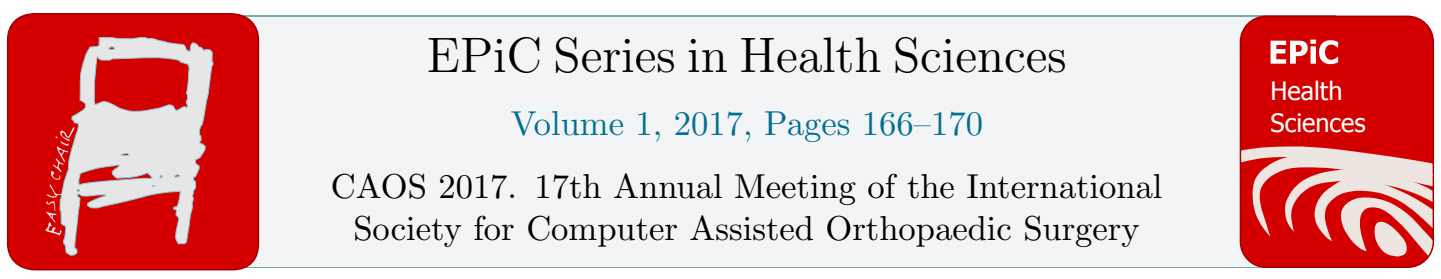

\title{
A Novel Tibiofemoral Kinematics Measurement System Based On Multi-Channel A-Mode Ultrasound System
}

\author{
Kenan Niu $^{1 *}$, Victor Sluiter ${ }^{1}$, André M.J. Sprengers ${ }^{1,2}$, Jasper Homminga ${ }^{1}$ \\ and Nico Verdonschot ${ }^{1,2}$ \\ ${ }^{1}$ University of Twente, Enschede, The Netherlands \\ ${ }^{2}$ Radboud University Medical Center, Nijmegen, The Netherlands \\ niukenan@gmail.com, V.I.Sluiter@utwente.nl, \\ andre.sprengers@radboudumc.nl, j.j.homminga@utwente.nl, \\ nico.verdonschoteradboudumc.nl
}

\begin{abstract}
Improving the accuracy of measuring 6 degree of freedom tibiofemoral kinematics is a crucial step in gait analysis. The skin-marker estimated kinematics is subject to soft tissue artefacts. Fluoroscopic systems have been reported to achieve high accurate kinematics, but induced irradiation and a limited field of view and its high cost hampers routine usage on large patient cohorts. The aim of this study is to assess the feasibility of measuring tibiofemoral kinematics using multi-channel A-mode ultrasound system in cadaver experiment and assess its achievable accuracy. A full cadaver was placed on the surgery table and legs were hanging in the air. Upper body was fixated and right leg was pulled by rope to generate a movement. To track the ground truth of the motion, two bone pins with optical markers were mounted to the femur and tibia separately. Six custom A-mode ultrasound holders containing $30 \mathrm{~A}$-mode ultrasound transducers and 18 optical markers were mounted on six anatomical regions. Calculating the bone to each ultrasound transducer distance from ultrasound echo, and using the spatial information of the optical markers on the holders, 30 points were determined. The segmented bone models acquired from CT or MRI were registered to the corresponding points. Then the tibiofemoral kinematics were determined from the positions of registered femur and tibia per frame. This study has presented a multi-channel A-mode ultrasound system and proven its feasibility of reconstructing tibiofemoral kinematics in cadaver experiment. Although the reconstructed tibiofemoral kinematics is less accurate than fluoroscopic system, it outperforms skin-mounted markers system. Thus, this Amode Ultrasound approach could provide a non-invasive and non-radiative method for
\end{abstract}

${ }^{*}$ Created the first draft of this document 
measuring tibiofemoral kinematics, which may be used in clinic gait analysis or even computer-aided orthopedic surgery.

\section{Introduction}

Improving the accuracy of measuring 6 degree of freedom (DOF) tibiofemoral kinematics is a crucial step in gait analysis. The use of skin-mounted markers in which the trajectories of skin markers represent the movements of the bony segments beneath the skin, is well established. However, the skin-marker estimated kinematics is subject to soft tissue artifacts (STA), resulting in errors up to $50 \mathrm{~mm}$ (Akbarshahi et al., 2010). In term of kinematics, an average error of $4.4^{\circ}$ and $13.1^{\circ}$ was found for the rotation angles and 13.0 and $16.1 \mathrm{~mm}$ for the translations for walk and cut respectively (Benoit et al., 2006). As an alternative to skin marker systems, fluoroscopic systems have been reported to achieve accuracies of kinematics in the order of $1 \mathrm{~mm}$ and 2 degrees (Guan, Gray, Keynejad, \& Pandy, 2016), but the induced irradiation to the subject, the limited field of view, and the high costs hamper routine usage on large patient cohorts. Ultrasound (US) technology could provide a solution; it has the potential to detect the bone boundaries through the soft tissue during movement. It has been demonstrated that registering US image to the segmented bone in computer-aided orthopaedic surgery (CAOS) (Barratt et al., 2006) as well as estimating tibiofemoral kinematics (Masum, Pickering, Lambert, Scarvell, \& Smith, 2014) are possible. In this study, we present a novel multi-channel A-mode ultrasound system and show the feasibility of measuring tibiofemoral kinematics in an in-vitro experiment and assess the achievable accuracy relative to the ground truth.

\section{Materials and methods}

A full cadaver was placed on the surgery table with its legs overhanging the edge of the table. Upper body was fixated to the table by nylon straps while the right leg was moved by means of a rope. To track the ground truth of the motion, two bone pins with optical markers were mounted to both the femur and tibia before CT scan. The bone models of femur and tibia were manually segmented (Mimics ${ }^{\circledR}$ 17.0) from CT image and the 3D locations of the optical markers were also digitized which provided the ground truth for all movement. Additionally, six custom holders containing 30 A-mode ultrasound transducers and 18 optical markers were mounted to six anatomical regions: ankle, middle tibia, tibia epicondyles, femur epicondyles, middle femur and greater trochanters (see figure 1-B).

All optical markers were tracked by a Visualeyez system (VZ4000z) operating at $60 \mathrm{~Hz}$. The ultrasound signal was captured and was synchronized with the Visualeyez system in the Diagnostic Sonar FI Toolbox. Custom written software (LabVIEW 2015) was used to process all data. By calculating the bone to ultrasound transducer distance from the ultrasound echo and using the spatial information of the optical markers on the holders, 30 bone surface points are determined. The sample rate of acquiring ultrasound points is $20 \mathrm{~Hz}$. During measurement, the acquired bone surface points were fed to a registration algorithm that combined an iterative closest point algorithm (Besl \& McKay, 1992) and perturbation method (Ma \& Ellis, 2003) to register corresponding bony segments to the acquired points for every timeframe. When the anatomical frames of femur and tibia were determined from segmented bone models (Miranda, Rainbow, Leventhal, Crisco, \& Fleming, 2010), the tibiofemoral kinematics were determined from the positions of the registered femur and tibia per timeframe (Grood \& Suntay, 1983). These estimated tibiofemoral kinematics were subsequently displayed in real-time. 


\section{Results}

The resulting kinematics showed an average error of $1.25 \pm 0.94^{\circ}$ (mean \pm standard deviation) for flexion-extension, $9.06 \pm 3.94^{\circ}$ for internal-external rotation, and $2.62 \pm 1.34^{\circ}$ for adductionabduction rotation. All three translation components had similar trends as the ground truth translations. There was an average error of $3.05 \pm 2.09 \mathrm{~mm}$ for tibia anterior-posterior translation, $5.25 \pm 2.90 \mathrm{~mm}$ for tibia distal-proximal translation, and $6.76 \pm 2.73 \mathrm{~mm}$ for tibia medial-lateral translation (see Figure 1-C).
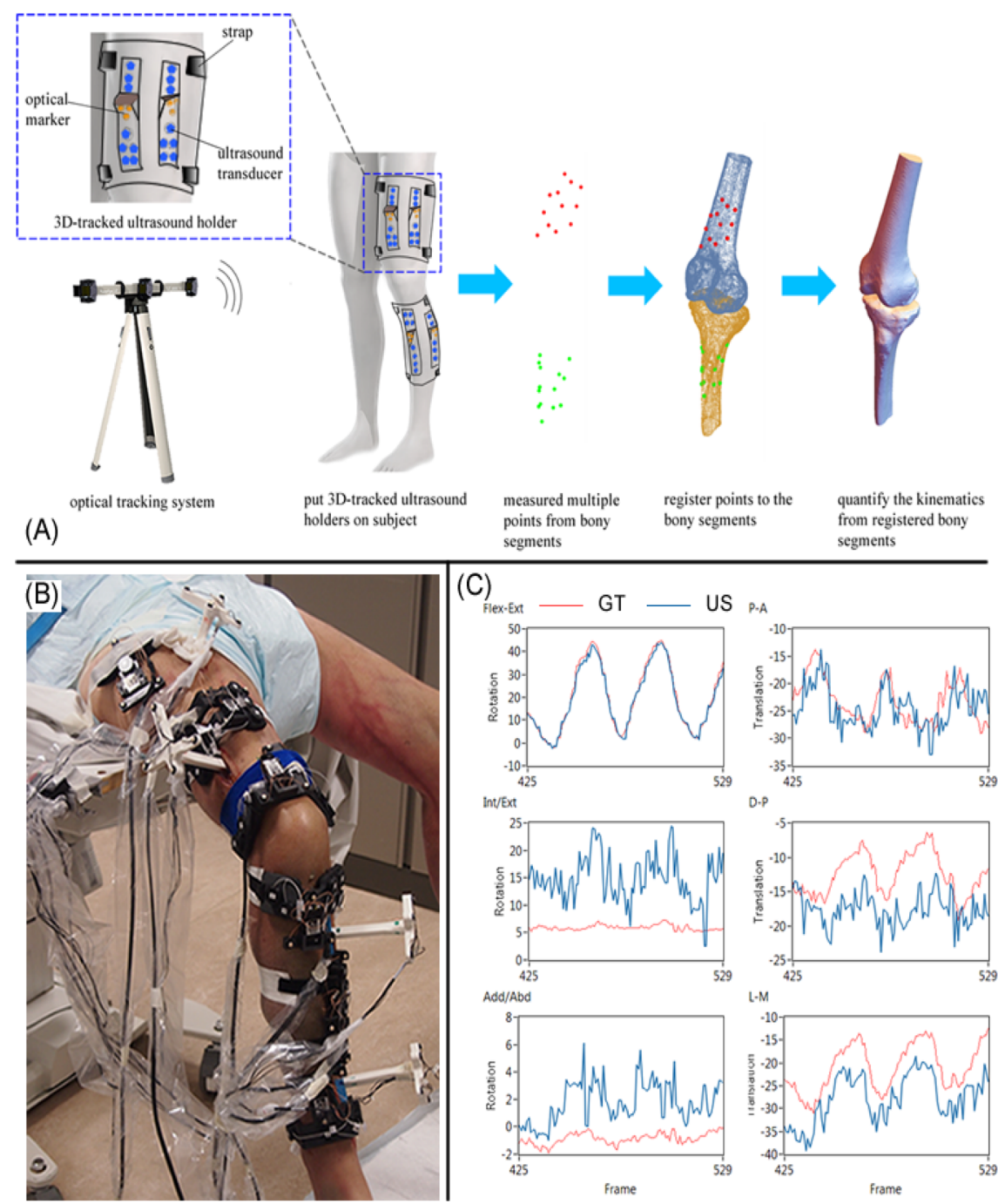

Figure 1 The schematic of multiple US knee kinematics tracking system; (B): the setup of in-vitro experiment with mounting 6 ultrasound holders (black color) and 4 bone pins (white color); (C): the estimated 6 DOF tibiofemoral kinematics (US) relative to ground truth (GT). 


\section{Discussion}

Compared to skin-mounted markers, multi-channel A-mode US system has the potential to provide more accurate tibiofemoral kinematics, with high accuracy in flexion-extension angles, adduction-abduction angles and tibia anterior-posterior translation.

However, internal-external angles and tibia distal-proximal translation and tibia medial-lateral translation show relatively high errors. These high errors can be caused by several factors. First, missing bone reflection point or incorrect reflection bone detected, both most prominent during dynamic movement. The incident angle of A-mode ultrasound transducer (Fieten et al., 2009; Heger et al., 2007) affects the quality of ultrasound echo. By keeping the A-mode ultrasound transducer perpendicular to the bone surface it will be easier to find the bone peak in echo (Mozes, Chang, Arata, $\&$ Zhao, 2010). However, this is difficult to implement in practice, during movement. The bone to transducer distance was determined from ultrasound echo. Sometimes the incorrect peak was chosen and then an wrong bone reflection point was used for registration. Hence, the future work will be the improvement of keeping the bone "in sight" of A-mode US during movement and the development of more accurate and reliable peak detection method. Second, inherent errors of optical tracking systems, that cannot be easily eliminated during experiments. Hence a systematic analysis of error model of optical system will help to understand how the error of each optical marker transmits to final ultrasound reflection points. Third, the lack of geometric constraints in distal-proximal direction and the lack of transducers covering more anatomical regions. In our study, we limited the number of Amode US transducers for the first prototype to thirty. In the future, more A-mode ultrasound transducers will be added and relocated on newly designed custom holders to cover more anatomical regions.

Although our system has a lower accuracy of reconstructed kinematics than a fluoroscopic system, it provides an non-invasive and non-irradiating approach to reconstruct tibiofemoral kinematics. With the improvements implemented in the future, the accuracy of tibiofemoral kinematics by multichannel A-mode US systems will have the potential to be comparable to that of fluoroscopic systems.

This study presented a multi-channel A-mode ultrasound system and showed its feasibility of reconstructing tibiofemoral kinematics in a cadaver experiment. The reconstructed tibiofemoral kinematics is less accurate than fluoroscopic system, but outperforms skin-marker systems. Thus, this multi-channel A-mode ultrasound system provides a non-invasive and non-irradiative method of measuring tibiofemoral kinematics, which may be used in clinic gait analysis or even computer-aided orthopaedic surgery.

\section{References}

Akbarshahi, M., Schache, A. G., Fernandez, J. W., Baker, R., Banks, S., \& Pandy, M. G. (2010). Noninvasive assessment of soft-tissue artifact and its effect on knee joint kinematics during functional activity. $J$ Biomech, 43(7), 1292-1301. doi:http://dx.doi.org/10.1016/j.jbiomech.2010.01.002

Barratt, D. C., Penney, G. P., Chan, C. S., Slomczykowski, M., Carter, T. J., Edwards, P. J., \& Hawkes, D. J. (2006). Self-calibrating 3D-ultrasound-based bone registration for minimally invasive orthopedic surgery. IEEE Trans Med Imaging, 25(3), 312-323. doi:10.1109/TMI.2005.862736

Benoit, D. L., Ramsey, D. K., Lamontagne, M., Xu, L., Wretenberg, P., \& Renström, P. (2006). Effect of skin movement artifact on knee kinematics during gait and cutting motions measured in vivo. Gait Posture, 24(2), 152-164. doi:http://dx.doi.org/10.1016/j.gaitpost.2005.04.012 
Besl, P. J., \& McKay, H. D. (1992). A method for registration of 3-D shapes. IEEE Trans Pattern Anal Mach Intell, 14(2), 239-256. doi:10.1109/34.121791

Fieten, L., Schmieder, K., Engelhardt, M., Pasalic, L., Radermacher, K., \& Heger, S. (2009). Fast and accurate registration of cranial CT images with A-mode ultrasound. Int J Comput Assist Radiol Surg, 4(3), 225-237. doi:10.1007/s11548-009-0288-z

Grood, E. S., \& Suntay, W. J. (1983). A joint coordinate system for the clinical description of threedimensional motions: application to the knee. J Biomech Eng, 105(2), 136-144.

Guan, S., Gray, H. A., Keynejad, F., \& Pandy, M. G. (2016). Mobile Biplane X-Ray Imaging System for Measuring 3D Dynamic Joint Motion During Overground Gait. IEEE Trans Med Imaging, 35(1), 326-336. doi:10.1109/TMI.2015.2473168

Heger, S., Mumme, T., Sellei, R., De La Fuente, M., Wirtz, D. C., \& Radermacher, K. (2007). Amode ultrasound-based intra-femoral bone cement detection and 3D reconstruction in RTHR. Comput Aided Surg, 12(3), 168-175. doi:10.3109/10929080701336132

Ma, B., \& Ellis, R. E. (2003). Robust registration for computer-integrated orthopedic surgery: laboratory validation and clinical experience. Med Image Anal, 7(3), 237-250. doi:10.1016/s1361-8415(02)00133-0

Masum, M. A., Pickering, M., Lambert, A., Scarvell, J., \& Smith, P. (2014). Accuracy assessment of Tri-plane B-mode ultrasound for non-invasive 3D kinematic analysis of knee joints. BioMedical Engineering OnLine, 13, 122. doi:10.1186/1475-925X-13-122

Miranda, D. L., Rainbow, M. J., Leventhal, E. L., Crisco, J. J., \& Fleming, B. C. (2010). Automatic determination of anatomical coordinate systems for three-dimensional bone models of the isolated human knee. $J$ Biomech, 43(8), 1623-1626. doi:http://dx.doi.org/10.1016/j.jbiomech.2010.01.036

Mozes, A., Chang, T. C., Arata, L., \& Zhao, W. (2010). Three-dimensional A-mode ultrasound calibration and registration for robotic orthopaedic knee surgery. Int J Med Robot, 6(1), 91101. doi: $10.1002 /$ rcs.294 